\title{
CURRICULUM Y DOBLE LÓGICA DE INSERCIÓN: LO UNIVERSITARIO Y LAS PRÁCTICAS PROFESIONALES
}

\author{
ROBERTO FOLLARI \\ Profesor de grado y posgrado en la Facultad de Ciencias Políticas de la Universidad \\ Nacional de Cuyo - Mendoza, Argentina \\ rfollari@fcp.uncu.edu.ar
}

\begin{abstract}
RESUMEN
La práctica intrauniversitaria y sus reglas autolegitimatorias, no tienen relación directa con las prácticas profesionales de quienes egresan de las universidades. De tal manera los planes de estudio pueden mantener sistemática disociación con las necesidaes profesionales, sin que ello implique disfunción alguna dentro de la actividad universitaria. Por cierto que la Universidad no tiene por qué responder mecánicamente a las demandas del mercado; pero sí debe asumir que la noción de crítica social debe resultar inmanente a las prácticas profesionales para las cuales se forma a los estudiantes. De lo contrario, se prepara a los mismos para el desempleo y la inadecuación laboral, o para un discurso que será abandonado en cuanto se inicie la actividad profesional.

PLAN DE ESTUDIOS - ENSEÑANZA SUPERIOR - PROFESIONALIZACIÓN - MERCADO DE TRABAJO
\end{abstract}

\begin{abstract}
THE CURRICULUM AND THE DOUBLE LOGIC OF INSERTION: UNIVERSITY STUDENTS AND PROFESSIONAL PRACTICES. Intra-university practice and its self-legitimizing rules have no direct relationship with the professional practices of university graduates. So, study plans may be systematically dissociated from professional needs, but this does not implyes any dysfunction within university activity. It is true that the University has no reason to respond mechanically to the demands of the market, but the institution must assume that the notion of social criticism is to result immanent to the professional practice for which it prepares students. Otherwise, it prepares them for unemployment and unsuitable employment, or for a discourse that will be abandoned as soon as they start their professional activities.

CURRICULUM - HIGHER EDUCATION - PROFISSIONALIZATION - LABOUR MARKET
\end{abstract}

Este artículo ha sido redactado para su presentación al Congreso Internacional de Educación: Curriculum, en la Universidad Autónoma de Tlaxcala, México, noviembre de 2009. 


\section{¿MITO DEL CURRICULUM?}

Fue precisamente en México, país donde la discusión especializada sobre educación superior ha alcanzado envergadura desde hace muchos años constituyendo todo un campo específico de análisis, que hay quien se ha referido al "mito" del curriculum. ¿Qué puede significar esto? (De Alba, 1991).

Por una parte, la inicial cuestión sobre la formalización curricular hacia los años 60 del siglo $X X$, ponía el acento en la planificación. Se trataba de diseñar un buen plan de estudios, con programas de cursos que le fueran coherentes. Con esto pareció bastar; luego la tarea docente debía ceñirse a ese patrón preestablecido. Se supuso que la realidad se adecuaría a esas exigencias ideales.

Pero la realidad es terca, y pronto se advirtió la insuficiencia de poner el acento sólo en el momento planificador. En Latinoamérica, las limitaciones de recursos y equipamiento, más las tradiciones culturales para las cuales el apego a determinismos no funciona, llevaron a que algunos teóricos se preguntaran desde EEUU - por qué "estamos fracasando" en Latinoamérical. Indagaban por qué cierto tecnocratismo formalizante que nos exigía saber desde el primer día de clase cuántas hojas de cuadernos los alumnos habrían utilizado el último día, no hallaría eco entre nosotros.

Además, en Latinoamérica, somos hijos de influencias centroeuropeas, de modo que al eficientismo crudo suele oponerse cierta incapacidad para lo pragmático, junto a una considerable tendencia de apego a lo teórico, a lo conceptual, que en el mundo sajón tiene menor cabida. Ello ha permitido una notable cantidad de contribuciones al análisis crítico de los procedimientos curriculares propuestos en los años 60. La crítica apuntó sobre todo a mostrar que la cuestión curricular iba más allá de una buena planificación, que había que considerar las cuestiones institucionales, la perspectiva del docente, incluso el contexto social y político ; el tema del curriculum hace a toda la situación en que se inscribe el trabajo académico.

I. Según lo planteado por los difusores estadounidenses de la Tecnología Educativa, tal el caso de Chadwick.

2. Han participado de ese debate, entre otros, Angel Díaz Barriga, Raquel Glazman, Alfredo Furlan, Alicia de Alba; también me fue dado personalmente tener lugar en el mismo. 
La crítica era pertinente, y ha sido sumamente fecunda para advertir que no se trata sólo de poner buenas previsiones en el papel, sino de considerar las reales condiciones en que pueden llevarse a cabo. Un plan de estudios adecuado es algo más que una planificación detallada: será también aquel que tenga en cuenta las efectivas posibilidades, que entusiasme a los alumnos, que interese a los futuros empleadores, que comprometa realmente a los docentes tanto en su conformación como en su aplicación concreta.

Este hallazgo fundamental de la crítica latinoamericana se acompañó de un efecto no querido: la "disolución de los límites" de la problemática curricular. Ya curriculum dejó de ser sinónimo de plan de estudios, y aún de aplicación de éste, para ocuparse de toda la acción académica desarrollada en las instituciones educativas. Lo curricular salió del encapsulamiento, pero también se volvió coextensivo (equivalente) de la entera actividad institucional. La problemática se extendió sin fijar límites.

Por esto se afirmó que podría haber mito en lo curricular. Aparecería como si fuera el espacio privilegiado desde el cual toda la problemática de lo educativo podría visualizarse.

Y por esta vía crítica se dio la paradoja de reforzar - involuntariamente - lo producido por la propuesta inicial ocupada de la formalización de la planificación curricular. Esta última, la conocemos todos. Cada vez que hay crisis en los resultados del trabajo de la institución, se proponen cambios en el plan de estudios. Mágica posibilidad, el plan de estudio nos salvará. Poco importa que los docentes no se sientan interpretados en él, que tales docentes sean exactamente los mismos que estaban antes pero con nuevas etiquetas en las materias asignadas, que el perfil profesional no se defina o que la coherencia entre cada curso y los demás (y la de cada uno con el conjunto) no se garantice. El plan de estudios aparece a menudo - aún hoy - como la receta universal, la posibilidad omnímoda, la nueva palanca habilitadora. "Dame un plan de estudio y moveré..."

Por esto, es importante que no nos engañemos. Todos conocemos las posibilidades, pero también debemos reconocer los límites del recurso al cambio de plan. Estemos atentos con un ejercicio elemental de lógica: un buen plan de estudios es condición indispensable para lograr resultados de aprendizaje deseables. Sin duda. Pero no es condición suficiente: sólo eso no basta. 


\section{EL PERFIL PROFESIONAL: COMPLEJIDADES NO-PENSADAS}

Asumida la cuestión de que lo curricular depende en su consecución de condiciones que están más allá del plan de estudios, veamos ahora la cuestión crucial del perfil profesional. De manera todavía germinal, es una cuestión que ya aparecía en los escritos iniciales sobre el tema. Un plan de estudios debe orientarse a las habilidades, capacidades, destrezas y conocimientos teóricos que son propios del campo profesional para el cual se está preparando. Hay que especificar el perfil profesional, y orientar a su consecución el plan de estudios. Tal perfil no puede ser un listado general de actividades posibles, sino un estudio del mercado laboral suficientemente serio para orientarse de acuerdo a las tendencias reales en la demanda social. Luego, habrá que fijar el modo en que cada materia del plan colabora específicamente a esto, sin pretender la cerrazón del acuerdo "punto a punto" propia de los tecnócratas, pero tampoco dejando a la improvisación el establecimiento de la cuestión. En la Argentina, cada profesor suele organizar su curso sin referencia alguna al perfil, lo cual redunda en fortalecer las fuertes disociaciones que existen entre lo que ofrece la Universidad y lo que luego se le exige al profesional en su desempeño. Todavía hoy muchos alumnos - desde Medicina a Sociología - sienten al recibirse, que no los prepararon exactamente para aquello en que deberán trabajar; a veces, simplemente no pueden especificar cuáles serían sus funciones en la sociedad. Se los formó pensando en los contenidos científicos de su disciplina, pero no en el ejercicio profesional de su rol.

Es cierto que no podemos abandonar la reflexión académica en aras de un pragmatismo miope, como el que predomina en estos tiempos: sin duda es imprescindible sostener la coherencia científica, y el valor de la teoría y de la discusión conceptual. Pero no olvidemos direccionarnos también al rol profesional, si no queremos quedar disociados de la demanda social y colaborar así a la futura desocupación de los profesionales, ya favorecida por razones económicas estructurales.

Si no queremos profesores guiando taxis, habrá que trabajar la cuestión de cómo se articula la formación del estudiante con la determinación del perfil.

Por cierto, esa adecuación del perfil a la práctica profesional no es algo tan obvio como parece. Estudios realizados hace ya tres décadas mostraron que la práctica de una profesión no es unívoca: puede haber médicos al servicio 
de actividad privada muy costosa para los usuarios, otros ubicados en centros asistenciales estatales de punta (ej.: Medicina Nuclear), otros en estatales donde concurren los sectores sociales más pobres. No hay práctica, sino diferenciadas prácticas profesionales; y por cierto que no resulta neutro formar prioritariamente para uno u otra (Follari, Berruezo, 1981; Guevara Niebla, 1976). Originalmente se las caracterizó como prácticas decadente, dominante y alternativa. Esta última cubriría la finalidad de servir a los sectores más desfavorecidos de la sociedad, como actividad compensatoria de desigualdades estructurales. Sin sobredimensionar este recurso (que no puede resolver, sino sólo paliar ese tipo de problemas), vale la pena intentar estudiar diferencialmente las diversas prácticas que se dan (o que podrían potencialmente darse) de una profesión, de manera que los estudiantes puedan aprender no sólo lo exigido por los empleadores más esperables, sino también por aquellos que tienen la necesidad social del servicio profesional, sin tener a menudo cómo acceder a él.

Es de destacar que esta discusión no se da actualmente en los países latinoamericanos más ligados a políticas neoliberales y sería de utilidad instalarla, más allá de los parámetros eficientistas que surgen desde las perspectivas más difundidas. Estas últimas, ligan los roles profesionales directamente a las necesidades empresariales, y suelen hacer desaparecer todo dejo crítico-social, cuando no toda arista teórica en la formación.

\section{Ciencia y profesión}

Pensemos más sobre la cuestión de profesiones y ciencias. Vale la pena especificar sus mutuas diferencias, pocas veces trabajadas. Del aprendizaje de una ciencia, no se sigue directamente la constitución de una profesión. Es el caso de la Física, o de la Sociología. Quien las ha aprendido, es un científico puro. Es decir, alguien que puede trabajar como docente e investigador en esa disciplina, pero que - de no hacerse mediaciones específicas- carece de competencias profesionales concretas de otro tipo.

Ello es inverso a lo que sucede con lo que podríamos denominar "profesiones que se han constituido como ciencias". Es decir, profesiones que han exigido para la habilitación de quienes las realizan, que éstos se formen científicamente en las universidades. En estos casos (tales como Comunicación social, Ingeniería e incluso Pedagogía), la condición se vuelve inversa. Hallamos profesiones me- 
dianamente bien definidas, que encuentran problemas epistemológicos para su constitución científica. Lo cual resulta coherente, pues no han surgido desde un desarrollo inmanente de la ciencia, lo cual las lleva a constituirse en torno de un objeto real más que de un objeto teórico, para retomar el conocido lenguaje del primer Bourdieu (Bourdieu et al., 1975).

En general, no hay suficiente advertencia de esta condición fuertemente diferencial entre un caso y el otro. De tal manera, los problemas que la formación universitaria muestra en cada caso son diferentes, aun cuando concurrentes en producir cierta ineficacia para lograr suficientes efectos en la práctica posterior del profesional.

Cabe primeramente señalar nuestro rechazo - ya manifestado - a la simple adecuación "punto a punto" de la universidad con las exigencias del mercado. Ello no es posible, ni tampoco deseable. La formación debe incluir aspectos conceptuales que vayan más allá de la demanda (sobre todo la demanda inmediata), de modo de trascender la característica exclusivamente actual de las necesidades del mercado laboral. Ello garantiza la capacidad de adecuación a modificaciones posteriores de las prácticas profesionales, a la vez que promueve la capacidad de autorreflexión siempre necesaria frente a las mismas.

De modo que abogamos por sostener el pensamiento crítico, la posibilidad de distancia en relación con las relaciones sociales expresamente existentes, para contribuir a hacer del profesional un intelectual, un analista simbólico que no quede atado a la inmediatez de la demanda.

Sin embargo, aquí no terminan los problemas, sino que apenas comienzan. Sucede que las prácticas que en su momento definimos como alternativas y emergentes no suelen estar ni suficientemente delineadas en la realidad del campo laboral efectivo, ni mucho menos en la definición de los planes de estudio.

Ello lleva a que - a menudo - los profesionales adscritos a profesiones con campo laboral relativamente definido, terminen realizando lo que plantean las prácticas dominantes, incluso si ha habido esfuerzos de formación en sentido contrario. Y la formación de las disciplinas científicas lleve a sus egresados directamente a la desocupación, o a la necesidad de trabajar en áreas ajenas a su formación. 


\section{El doble régimen de legitimación}

El problema de la disociación entre el campo laboral y lo ofrecido por las universidades puede entenderse en términos de un doble régimen legitimatorio.Para funcionar con éxito en la universidad, no se requiere que los egresados tengan éxito en su inserción laboral. Si bien agencias evaluadoras pretenden medir esta inserción, es notorio que la calidad investigativa de una universidad, por ejemplo, poco tiene que ver con la situación laboral de los egresados. La calidad científica de una institución universitaria no tiene relación directa (aunque sí la tiene mediada) con las posibilidades laborales de los estudiantes, en tanto futuros profesionales.

Digámoslo de una vez: aquello que permite que un docente sea aceptado dentro de su práctica universitaria, nada tiene que ver - las más de las veces - con las destrezas necesarias para la profesión del caso. Los alumnos no tienen, muchas veces, más que una vaga idea de cuáles sean esas destrezas y necesidades. De tal manera, un buen docente es aquel que mejor lo sea "en términos puramente universitarios".

Los docentes no estamos urgidos por la condición de los egresados de la universidad. Habitualmente ellos no están organizados como tales, y no hacen reclamos a la institución desde su rol profesional: se han desperdigado tras haber recibido su título habilitante, y en todo caso - a través de los colegios profesionales - sus reclamos van dirigidos al campo laboral, sólo rara vez al de la formación previa.

De modo que nos encontramos con la escisión entre "dos mundos". De una parte la universidad, donde los criterios de aceptabilidad pasan por la calidad académica o la aptitud docente, por la capacidad teórica y el pensamiento crítico; de la otra el espacio profesional, donde a menudo la adaptación al mercado laboral exige nula capacidad crítica, escasa aptitud para la teoría, y donde la capacidad académica o docente no vienen en absoluto a cuento.

De manera que el alumno está en la universidad en un espacio específico, y en el mundo laboral en otro, absolutamente diferentes desde el punto de vista de qué se requiere para tener éxito en cada uno de ellos. Lo cual conlleva una serie de efectos perversos (es decir, efectos no queridos) de la formación universitaria en relación con las prácticas profesionales de los egresados. 
Pensemos en la formación en ciencias básicas. Estas, definidamente, no son profesiones, pero ello no siempre es claro para quien comienza esas carreras; de algún modo se ofrecen como si fuesen profesiones, pues se supone que un físico o un sociólogo son personas que egresan con la posibilidad de un trabajo que tenga ciertos efectos sociales. Al menos, no es obvio que se niegue tal posibilidad y se piense solamente en formar académicos; esto se da sólo en algunos casos-límite, como literatura o matemáticas, donde lo extraacadémico tiene muy escaso campo de posibilidades ${ }^{3}$.

¿Qué sucede si se sigue "espontáneamente" esta composición epistémica del conocimiento en ciencias básicas? Que se forma a los estudiantes en el aprendizaje de teorías, desgajadas de cualquier uso social. Ello es funcional al espíritu intrauniversitario: la calidad explicativa aparece como el criterio de aceptabilidad de los discursos y prácticas, sin que medie la necesidad incómoda de ponerla en relación con problemas sociales urgentes, con investigaciones de campo, o con usos que vayan más allá de la lectura y análisis de textos (sin que neguemos el valor de los mismos; el "ir a la realidad" sin pasar por la lectura sistemática, es obviamente un craso error empirista contra el cual estamos totalmente instalados ${ }^{4}$ ).

De tal manera, la mejor carrera en una universidad suele ser la que es más intraacadémica; es aquella que mejor satisface los "criterios internos" de la institución en términos de validez cognitiva, de calidad de explicación, de definición conceptual de su objeto. En fin, es aquella que remite a una ciencia epistemológicamente mejor constituida. Por ello, en Argentina, el Consejo Nacional de Investigaciones Científicas y Técnicas - Conicet - tiene una comisión dedicada a Sociología, pero no la tiene en relación con Comunicación. El contraste entre ambas disciplinas es claro: podría decirse, simplificando, que la primera es una ciencia traspuesta (incómodamente) a profesión, y la segunda es una profesión - o cúmulo de las mismas - traspuesta(s) (incómodamente) a ciencia.

3. Aunque el aporte a lo operativo en esos casos no sea simplemente nulo; por ejemplo, la utilidad de lo literario para publicidad, o de la matemática para las estadísticas.

4. Conferir la obra de Bachelard, donde se destaca el peso constitutivo de lo teórico en referencia a lo empírico. 
De tal manera, la Sociología tiene un claro status superior dentro de las universidades. Se advierte incluso en su muy menor cantidad de alumnos ${ }^{5}$; éstos son más seleccionados, tienen un mayor interés propiamente intelectual y suelen ser concientes de la mejor definición cognoscitiva de su disciplina.

Los estudiantes de Comunicación triplican o cuadriplican en número a los anteriores, no tienen - en su mayoría - intereses intelectuales desgajados de los directamente laborales, e incluso, en no pocos casos, ligan su motivación profesional a la del éxito mediático y la notoriedad que éste asegura.

Lo curioso es cómo se invierte esta situación en relación con el momento de conseguir trabajo. Las posibilidades de empleo son enormemente mayores para los comunicadores, los cuales a su vez, si asumen actividades independientes de consultoría, tienen mejores horizontes y posibilidad de contratos que los sociólogos que intentan una actividad homóloga.

Esto que afirmamos suena plenamente consistente con lo antedicho: es obvio que si la comunicación nació como demanda desde el mundo de las profesiones hacia la universidad, tiene mejores condiciones laborales que aquellos que surgieron desde una necesidad universitaria, y luego la traspusieron hacia una profesión, necesariamente difusa.

Cierto fracaso universitario se advierte en ambos casos, al menos si nos limitamos al caso de la Universidad Nacional de Cuyo, donde me toca trabajar habitualmente. ¿En qué consiste el mismo? En el caso de los sociólogos, la advertencia de que lo que estudiaron en la universidad carece de aplicabilidad fuera de la misma. La calidad teórica y el pensamiento crítico se han presentado desgajados de usos sociales efectivos, de modo que están escindidos de posibilidades laborales, ya sea porque los egresados no fueron capacitados suficientemente, o porque desde la sociedad hay desconocimiento respecto de qué pudieran esperar de los mismos.

Las citas de los teóricos de las grandes líneas sociológicas, las abjuraciones del capitalismo - con todo lo que tienen de justificadas por las recurrentes

5. Tomo para el caso mi conocimiento de lo que sucede en la Facultad de Ciencias Políticas y Sociales de la Universidad Nacional de Cuyo (Mendoza, Argentina), donde se dictan esas dos carreras, además de licenciaturas en Ciencias Políticas y Administración, por una parte, y Trabajo Social, por la otra. Es de destacar que la información que cuento de otros países latinoamericanos es coincidente, en cuanto a la proporción de número de estudiantes entre Sociología y Comunicación. 
consecuencias desastrosas del mismo para nuestras sociedades ${ }^{6}$-, los conocimientos detallados sobre las discusiones científico-teóricas, las distinciones sofisticadas sobre causación estructural o determinación en última instancia, no pueden tener uso profesional sin alguna mediación adicional, la que notoriamente no se ha producido. Los egresados encuentran súbitamente que todo los que los justificaba como alumnos valiosos, no funciona para ser profesionales. Han sido ubicados como profesionales en un campo desconocido, y las características del mismo resultaban insospechadas desde la práctica universitaria.

En el caso de los egresados de Comunicación, a menudo se concilian con el mercado de una manera sorprendente. Alumnos que han aprendido la crítica del poder mediático (aunque en Comunicación a veces, y no casualmente, se imponen líneas teóricas que soslayan tal crítica $^{7}$ ), que están acostumbrados a saber sobre la concentración de la propiedad de los medios y la miseria intelectual que rige a muchos de los mismos, que han aprendido en el aula y también en los pasillos las posiciones de izquierda, no siempre pero sí frecuentemente se comportan luego en los medios como obedientes exponentes de la lógica mediática. Algunos pierden toda capacidad crítica en escaso tiempo, desnudando que, en realidad, ellos no adherían a un contenido valorativo específico como el de la universidad, sino a un principio más general: "A donde fueres, haz lo que vieres".

De tal manera, el principio lleva a quedar bien con aquello que en cada sitio se reclama. Si en la universidad queda bien el discurso crítico, tendremos discurso crítico; si en el mundo mediático se impone el discurso facilista y proempresarial, cuando allí estemos, haremos discurso proempresarial.

Se da la tentación de hacer una lectura puramente eticista-subjetiva de estas decisiones cambiantes, y echar la culpa a la falta de conciencia de los egresados o de los jóvenes actuales en general, a su descompromiso posmoderno, o al poder de la ideología dominante. Razones todas estas que no son

6. No es necesario abundar sobre las condiciones de marginación y desigualdad social que jalonan la historia de Latinoamérica, y que mayoritariamente se mantienen hoy, a pesar de parciales mejoras logradas con gobiernos populistas y progresistas hoy existentes en la región (desde Chávez a Evo Morales, o el Frente Amplio uruguayo).

7. Es el caso de los "estudios culturales" promovidos en Latinoamérica por Jesús Martín-Barbero y Néstor García Canclini. Para la crítica de su abandono de los medios como objeto de análisis, ver Reynoso (1980) e Follari (2002). 
falsas ni dejan de ser pertinentes, pero que no ponen el acento en lo central: si ello ocurre, algo erróneo está sucediendo con la formación que se ofrece a los alumnos en la educación superior.

\section{El reino de Universilandia}

¿Dónde estaría el problema? Ya nos referimos a la ingenuidad de suponer una total adecuación mutua entre oferta universitaria y demanda laboral, según la conocida utopía del pensamiento tecnocrático. Hemos apuntado que ni se puede, ni se debiera. Por diversas razones; una de ellas es la imposibilidad de borrar de un plumazo la diferencialidad de cultura institucional entre la universidad y las instancias laborales, especialmente las empresariales.

Conocemos una de las respuestas socorridas desde el establishment frente a esta situación: eliminemos la universidad, y produzcamos el conocimiento, en su conjunto, dentro de la empresa misma. Esta pretensión extremadamente reductiva tiene sus profetas, y es decisivo defender a la ciencia de su conversión en pura aplicación, y al pensamiento de su metamorfosis en pura tecnología y criterios de aplicabilidad, cuando no de exclusiva ganancia ${ }^{8}$.

La universidad mantiene la posibilidad de una autorreflexión social no urgida, y sirve de contrapeso - aunque inevitablemente débil - de las tendencias mercantiles que se imponen en el capitalismo, y de las utilitarias que pueden imponerse en cualquier tipo de organización social. De tal manera, es necesario defenderla activamente de aquellos que imaginan eliminarla, soñando por esa vía con el final del pensamiento crítico; y, a su vez, es necesario salvar a la ciencia de su reducción pura a tecnología, con lo cual cada vez se explicaría menos en aras de la sola aplicación, y se correría el riesgo de una gradual desaparición de la ciencia; tal sería el caso si la universidad desapareciera, y el conocimiento se centrara en las empresas.

Asumido lo anterior, no se sigue de lo mismo que cuanto más lejos esté la universidad de usos sociales del conocimiento, mejor será. De ningún modo es así. Por supuesto que cabrá establecer cuáles son esos "usos sociales", pero la distancia radical entre mundo académico y prácticas sociales externas a la universidad es la que conlleva los fracasos en la formación de que venimos

8. Ver la posición proempresarial en Gibbons (1997). 
hablando más arriba, ejemplificados en este caso en las carreras de Sociología y Comunicación.

Tomemos el primer caso. Lo malo de la formación no es la apelación a la teoría, ni tampoco la asunción del pensamiento crítico. Por el contrario, lamentable sería que las universidades fueran eco del peor sentido común que sigue la tónica de lo establecido, y que se muestra incapaz de criticar lo existente.

Lo problemático es, sin embargo, la incapacidad para ligar teoría y crítica con formas de investigación y de práctica social concreta. Algo que exige mucho más que la sola, y por demás necesaria, remisión a los libros y autores. A partir de tales lecturas, lo necesario sería que los estudiantes - pensemos por ahora en Sociología - realizaran esas prácticas dentro del desarrollo de su plan de estudios. Es decir, que se pudiera mostrar que se estudia los temas hoy pertinentes: seguridad ciudadana, narcotráfico, peso identitario y económico de las actividades deportivas rentadas, cultura del rock y de la música en general, turismo, adhesiones políticas etc. Todos estos temas debieran ser empírica y concretamente investigados por los docentes, con auxilio y participación de los estudiantes durante su formación.

Ello tendría resultados en varios frentes. Por un lado, se reconocería socialmente la existencia de la profesión, es decir, se la haría conocer en concreto. Por otro, los estudiantes aprenderían las destrezas necesarias, tanto de investigación como de intervención. También se cubriría funciones que la sociedad efectivamente necesita y a menudo están vacantes y, sobre todo, se reubicaría la noción de pensamiento crítico. Éste no sería concebido como una suerte de condición de "exterioridad" en relación al concreto mundo de las relaciones sociales tal cual están dadas, sino como una especial manera de estar dentro de las mismas. Es decir - en el mejor sentido del pensamiento dialéctico - la negatividad es interna a lo que niega, y ser adversario del capitalismo no es ponerse fuera del mismo (en un espacio a menudo sólo imaginario), sino habitar en él promoviendo las posibilidades de su cambio (Parisi, 1979).

Lo mismo, aunque desde un ángulo diferente, puede señalarse sobre los egresados de Comunicación. Lo que ha sucedido en su formación, es que no se ha reflexionado suficientemente sobre lo cambiante del espacio de la universidad con el de los medios, donde la mayoría trabajará luego (ello es extensible a otros ámbitos laborales, como las empresas en el caso de los "comunicadores institu- 
cionales"). Los alumnos realizan pasantías en sus planes de estudio, pero ellas no revierten en reflexión sistemática concomitante y posterior; mucho menos, en una puesta en común colectiva que compare y sopese diferentes experiencias. De tal modo, la universidad no prepara al estudiante para el choque con la realidad del medio, y no se discute lo suficiente sobre las enormes diferencias de criterios que rigen la actividad en un espacio y el otro.

Por supuesto que no somos ilusos ni delirantes como para esperar de los egresados que se comporten en los medios como si estuvieran en la universidad. Ello no sería beneficioso para nadie, e implicaría conflictos irresolubles y permanentes para el egresado. De lo que se trata es de que la marca de la universidad no se pierda, que exista precisamente capacidad crítica para ofrecer un producto donde no todo sea solamente la expresión dominante, en el cual algún rasgo de conocimiento, inteligencia teórica o conciencia crítica pueda deslizarse en los mensajes mediáticos en que el egresado interviene.

De tal modo, lejos estamos de imaginar que mágicamente la universidad pueda por sí sola superar el condicionamiento que a los egresados hace el tipo de legitimidad que promueve el medio, que es totalmente diferente del de la universidad. Pero sí creemos que la institución educativa puede hacer más de lo que hace; y ello tiene que ver con exponer la formación a lo que sucede en el espacio mediático, con investigarlo más y relacionarse efectivamente con el mismo desde la universidad, de manera que, cuando se llegue a la titulación, no exista un salto desde lo conocido a lo desconocido, y desde un tipo de discurso a otro por completo advertido como nuevo y diferente.

Advertimos que, en ambos casos, el problema es el mismo: la universidad está cerrada sobre sí, sus planes de estudio no incluyen suficientemente la relación con las realidades socialmente relevantes. En el caso de Comunicación, sostener prácticas con sectores populares y medios alternativos sería sumamente útil, de modo que los egresados advirtieran que existen posibilidades más gratificantes y socialmente necesarias que la instalación y el empleo en los grandes medios, con su lógica del lucro y del servicio casi unánime a la ideología dominante.

Aquí nos reencontramos con lo ya dicho sobre "el mito del currículo". Esto no se modifica ni soluciona sólo con cambiar los planes de estudio. La "lejanía" de la universidad en relación con otros espacios sociales proviene de raíces organizativas profundas, y no puede ser superada con simples apelaciones voluntaristas. 
Bien se lo ha estudiado: la universidad es una institución "pesada en la base", una "anarquía organizada" (Krotsch, 1994). Es decir, un espacio que no tiene organización centralizada, donde la jefatura administrativo-gestional no se corresponde con la jerarquía académica - llevando a desconocimientos desde cada una de ambas partes hacia la legitimidad de la otra -, donde cada actor está ligado a "su" propia carrera que a menudo tiene como referente a la comunidad académica disciplinaria y no a la institución, donde toda noción de la institución como conjunto suele ser borrosa, cuando no inexistente.

Esta peculiaridad de las universidades no es simplemente un defecto; así lo requiere el trabajo intelectual, que difícilmente pudiera ser regimentado por criterios racional-burocráticos del tipo de los de una oficina empresarial o de gobierno.

Pero dicho lo anterior, es notorio que la universidad se convierte en un mundo en sí mismo, aislada del resto de lo social. Sólo allí existen, por ejemplo, ciertos grupos de ultraizquierda de incierto o inexistente peso en sectores obreros o campesinos; sólo allí el pensamiento puede tener como objeto de análisis al pensamiento mismo, y configurar una cierta endogamia que se alimenta permanentemente de su propia producción.

De tal manera, es claro que lo que permite a los docentes e investigadores reproducirse como tales, e incluso escalar en el campo académico ${ }^{\text {, }}$ poco o nada tiene que ver con las posibilidades laborales de los egresados. El horizonte de inteligibilidad de la actividad de estos últimos puede ser por completo dejado de lado por los docentes. Estos están inmersos en su lucha dentro del campo, y los egresados corresponden a otros campos, no al propio (los titulados pertenecerán a campos profesionales, no académicos). Estos no inciden en las prácticas de los universitarios, no llevan puntaje a sus evaluaciones docentes, no figuran a la hora de la cualificación de su actividad. Ni siquiera, habitualmente, el singular aporte de cada docente para el futuro trabajo de los alumnos incide en el juicio de los alumnos sobre los mismos, en la medida en que los estudiantes aún no se advierten como egresados futuros, sino sólo como "alumnos presentes".

De tal modo, ser "buen universitario", nada tiene que ver con ser alguien que se prepare para el mundo del trabajo posterior. La universidad

9. Acorde lá noción de "campo" producida por Bourdieu (2000). 
tiene sus propias reglas de legitimación, y ubicarse dentro de las mismas rara vez implica propiciar consecuencias importantes hacia la práctica posterior de los egresados.

No abundemos sobre otros aspectos de la cuestión: el aislamiento de cada docente (mayor en países como Argentina, donde subsiste la arcaica organización académica por cátedras), que hace que cada uno de los mismos pueda desconocer el plan de estudios, pues sólo da razón de su propio curso. La competitividad entre los profesores, a veces despiadada (Follari, 2008), que hace inviable la necesaria cooperación que dé al plan de estudios - no sólo en los papeles sino en su ejercicio - coherencia de conjunto. La falta de peso de las autoridades por esa "pesantez en la base", que lleva a desatender las recomendaciones, o aun las órdenes, de directores de carreras. Y, por último, algo que ya hemos adelantado: el hecho de que no veremos las caras de los egresados; los docentes sólo atendemos estudiantes, y no necesitamos hacernos cargo del resultado laboral de nuestra enseñanza.

\section{Tendiendo puentes}

No es nada fácil revertir la realidad que planteamos. Por ahora, nos interesa dejarla plasmada para empezar a reflexionar sobre la misma, y apuntar sólo algunas líneas posibles para ir avanzando.

Las universidades no van a modificar radicalmente sus modelos organizativos; incluso podrían hacerlo en sentido tecnocrático-empresarial, que ha venido sucediendo desde las políticas neoliberales de los años 80 (Aboites, 2009), y esto iría exactamente en dirección contraria de la que estamos sugiriendo.

No estamos contra la teoría y la crítica propias de las universidades, sino por una modificación de sus modalidades. Por una puesta en práctica de las mismas en investigaciones e intervenciones socialmente pertinentes, que puedan dar realce social a la universidad, y mostrar su pertinencia desde los intereses mayoritarios.

Sabemos que nada de ello es fácil, que en convenios con instituciones empresariales, gubernamentales, mediáticas y aún sociales en general, se cruzan lógicas diversas, y que la tensión entre las mismas hace incómoda la actividad para los universitarios (por cierto, también para los demás respecto de ellos), de modo que la tendencia es a "quedarse en casa" y manejarse con lo previamente conocido. 
Advertimos también que las modalidades organizativas de las universidades tienden a la laxitud, y que no puede imaginarse que las mismas se transfieran súbitamente hacia un orden más cerrado o jerárquico, que no es propio del mundo académico. La libertad de pensamiento exige libertad institucional, y ello es parte de la "anarquía organizada" en que nos toca movernos.

Pero ello no debiera conducir a la resignación o al quietismo. Habrá que pensar con fuerza en incluir, en la planificación misma del currículo, prácticas permanentes de investigación e intervención fuera de la universidad, que permitan ir viviendo ese "doble espacio" legitimatorio de universidad y sociedad externa desde el momento de ser estudiantes, para que los alumnos puedan desde entonces ir calibrando las características de la profesión, y de hecho ir aprendiéndolas activamente.

Y, sobre todo, habrá que hacer cierta reducción de los márgenes de anarquía institucional. La universidad no es una oficina, pero no tiene por qué ser un caos. Es imprescindible reforzar, en lo vivido y lo simbólico, la identidad institucional de universidades en general y carreras en particular. Hay que impulsar acciones en ese sentido, que permitan que en el plano de "lo vivido" - lo directamente experimentado - sintamos que en la universidad formamos parte de un colectivo, y que nuestra suerte no tiene sólo una significación individual; para ello, son útiles las evaluaciones por carrera o por departamento, que estimulen la producción como colectivo, y no sólo la personal que a menudo es incentivada (Díaz Barriga, 1997; Follari, 2002).

En ese sentido, la insistencia para promover proyectos en común entre varios docentes - ya sea de investigación, de intervención, o de ambas en conjunto - debe ser sostenida, así como también el conocimiento de la condición comparativa de la propia carrera con otras de la misma especialidad de otras universidades, o con las de otras especialidades dentro de la misma institución, con la finalidad de advertir si hay superación o caída en términos comparativos con ellas en diferentes momentos (por ejemplo, en número de investigaciones, de proyectos de intervención, tasa de retención de estudiantes, modos de reconocimiento público, proyectos socialmente útiles a sectores populares, etc.). No se requiere explayarse sobre la función motivacional de estas comparaciones dentro de parámetros colectivos, es decir, superadores de la lógica de rendimiento de cada docente individual. 
Hay una transformación por hacer, y las condiciones no son fáciles. No encontramos que pueda llegarse tan lejos como supone Boaventura de Sousa Santos (2004), con su habitual búsqueda proactiva del cambio; nos parece en gran medida deseable, pero difícilmente posible, al menos dentro del horizonte de visibilidad del presente. Pero sin dudas que parte del espíritu de su propuesta es el que aquí campea: sacar a la universidad de su autolegitimación interna para ponerla de cara a la sociedad y a sus prácticas habituales. Allí es donde la crítica alcanza más pleno sentido, no en la insularidad de las aulas. Y allí es donde, luego, nuestros alumnos transformados en egresados podrán encontrar, entonces, un suelo menos desconocido y menos hostil.

\section{BIBLIOGRAFÍA}

ABOITES, H. A Privatização da educação superior no México: novas e velhas realidades. Buenos Aires: Clacso, 2009. (bibliografía del curso Universidad y políticas de Estado: Alternativas a la Mercantilización de la Educación Superior: versión electrónica)

BOURDIEU, P. Intelectuales, política y poder. Buenos Aires: Eudeba, 2000.

BOURDIEU, P. et al. El Oficio de sociólogo. Buenos: Aires Siglo XXI, 1975.

DE ALBA, A. Curriculum: crisis, mito y perspectivas. México: Cesu/Unam, México, 1991.

DÍAZ BARRIGA, A. La Comunidad académica de la Unam ante los programas de estímulos al rendimiento. In: DÍAZ BARRIGA, A.; PACHECO, T. (Coord.). Universitarios: institucionalización académica y evaluación. México: Cesu/Unam, 1997. p.37-52.

FOLLARI, R. Modificaciones epistemológicas actuales, oferta educativa y planificación curricular. Revista Educação, Porto Alegre, v. I 8, n. 56, p. I 7I- | 83, mayo/ago. 2005.

. Problemas de evaluación de la universidad, y de evaluación de la investigación: perspectivas a partir del caso argentino. In: CASANOVA CARDIEL, H. Nuevas políticas de la educación superior. Coruña: Netbiblo, 2002. p.265-312.

- La Selva académica: los silenciados laberintos de los intelectuales en la universidad. Rosario: Homo Sapiens, 2008.

. Teorías débiles: para una crítica de la deconstrucción y de los estudios culturales. Rosario: Homo Sapiens, 2002.

FOLLARI, R.; BERRUEZO, J. Criterios e instrumentos para el diseño de planes de estudio. Revista Latinoamericana de Estudios Educativos, México, v. I I, n. I, p. I64-166, I98I. 
GIBBONS, M. (Comp.). La Nueva producción del conocimiento: la dinámica de la ciencia y la investigación en las sociedades contemporáneas. Barcelona: Pomares-Corredor, 1997.

GUEVARA NIEBLA, G. El Diseño curricular. México: Unam/Xochimilco, 1976.

KROTSCH, P. Organización, gobierno y evaluación universitaria. In: $\mathrm{KROTSCH}, \mathrm{P}$; PUIGGRÓS, A. (Comp.). Universidad y evaluación: estado del debate. Buenos Aires: Aique/Rei/ldeas, 1994.

PARISI, A. Filosofia y dialéctica. México: Edicol, 1979.

REYNOSO, C. Apogeo y decadencia de los estudios culturales. Barcelona: Gedisa, 1980.

SANTOS, B. S. A Universidade no século XXI: para uma reforma democrática e emancipatória da universidade. São Paulo: Cortez, 2004.

Recebido em: dezembro 2009

Aprovado para publicação em: fevereiro 2010 